\title{
Two-hybrid analysis of Ty3 capsid subdomain interactions
}

\author{
Min Zhang ${ }^{+1}$, Liza SZ Larsen+1, Becky Irwin', Virginia Bilanchone1 and Suzanne Sandmeyer*1,2,3
}

\begin{abstract}
Background: The yeast retrotransposon Ty3 forms stable virus-like particles. Gag3, the major structural protein, is composed of capsid, spacer and nucleocapsid domains. The capsid domain of Gag3 was previously modeled as a structure similar to retrovirus capsid.

Findings: Two-hybrid analysis was used to understand the interactions that contribute to particle assembly. Gag3 interacted with itself as predicted based on its role as the major structural protein. The N-terminal subdomain (NTD) of the capsid was able to interact with itself and with the C-terminal subdomain (CTD) of the capsid, but interacted less well with intact Gag3. Mutations previously shown to block particle assembly disrupted Gag3 interactions more than subdomain interactions.

Conclusions: The findings that the NTD interacts with itself and with the CTD are consistent with previous modeling and a role similar to that of the capsid in retrovirus particle structure. These results are consistent with a model in which the Gag3-Gag3 interactions that initiate assembly differ from the subdomain interactions that potentially underlie particle stability.
\end{abstract}

\section{Findings}

The Ty3 retrotransposon in budding yeast forms viruslike particles (VLPs) comprised of precursor Gag3 and Gag3-Pol3 polyproteins [1,2]. Previous alanine-scanning mutagenesis indicated that the $\mathrm{N}$-terminal domain (NTD) of the structural polyprotein Gag3 plays an important role in VLP formation [3]. During maturation, Gag3 is processed into $24 \mathrm{kDa}$ capsid (CA), $27 \mathrm{kDa}$ CA-spacer (SP), $3 \mathrm{kDa} \mathrm{SP}$, and $7 \mathrm{kDa}$ nucleocapsid (NC) protein by the Ty3 protease. Unlike most retrovirus cores, these cytoplasmic particles remain stable after proteolytic maturation.

Two-hybrid analysis [4] was used to better understand the contributions of Gag3 subdomains to formation and stability of the Ty3 VLP. Fusions of Gag3 and derivatives to the C-terminus of the Gal4-BD tagged with c-Myc were expressed from the high-copy, TRP1-marked pGBK vector (Clontech, Palo Alto, CA, USA). Fusions of Gag3 and derivatives to the $\mathrm{C}$-terminus of the Gal4-AD tagged with HA were expressed from the LEU2-marked high-

\footnotetext{
* Correspondence: sbsandme@uci.edu

${ }^{1}$ Department of Biological Chemistry, University of California, Irvine, CA, USA + Contributed equally

Full list of author information is available at the end of the article
}

copy plasmid pGAD T7 (pGAD). These fusions were constructed by amplifying the appropriate regions from Ty3 Gag3 subclones in pGEM (Invitrogen, Carlsbad, CA, USA) using polymerase chain reaction (PCR) primers containing NdeI and BamHI sites at the $5^{\prime}$ and $3^{\prime}$ outside ends, respectively and ligating fragments to the pGBK and pGAD vectors linearized with NdeI and BamHI. Constructs (Table 1) were confirmed by DNA sequence analysis (GeneWiz, South Plainfield, NJ, USA). Fusion proteins in these vectors are expressed under the constitutive $A D H 1$ promoter. Two-hybrid plasmids and negative control vector plasmids were transformed into yeast strain yAH109 (MATa trp1-901 leu2-3, 112 ura3-52 his3200 gal4A gal80A LYS2::GAL1 $1_{\text {UAS/TATA }}$ HIS3 GAL2 ${ }_{\text {UAS/ }}$ TATA $-A D E 2, \quad U R A 3:: M E L 1_{\text {UAS/TATA }}$-lacZ), which has $A D E 2, H I S 3, L a c Z$, and MEL1 reporters regulated by the GAL4 responsive upstream activating sequences (UASs) (Clontech). Preliminary tests showed that expression of $A D E 2$, which results in cream-colored colonies and growth in medium lacking adenine, provided the most reliable detection of two-hybrid interaction (data not shown). The yAH109 transformants containing pairwise combinations of pGAD and pGBK plasmids were selected on medium lacking tryptophan and leucine. Four 
Table 1: Two-hybrid plasmids

\begin{tabular}{|c|c|c|c|}
\hline Vector/plasmid & Ndel/BamHI insert & Gag3 residues & Mutation(s) \\
\hline \multicolumn{4}{|c|}{ pGBK T7 (with Gal4-BD): } \\
\hline pMZ2667 & Gag3 & $1-290$ & WT \\
\hline pMZ2668 & Gag3 $\Delta S P$ & $\Delta 207-233$ & WT \\
\hline pMZ2669 & $\mathrm{p} 27$ & $1-233$ & WT \\
\hline pMZ2670 & $C A$ & $1-207$ & WT \\
\hline pMZ2671 & CA-NTD & $1-135$ & WT \\
\hline pMZ2672 & CA-CTD & $136-207$ & WT \\
\hline pMZ2673 & NC & $234-290$ & WT \\
\hline pMZ2676 & Gag3 & $1-290$ & D60A/R63A (M4) \\
\hline pMZ2652 & Gag3 & $1-290$ & $\mathrm{E} 148 \mathrm{~A} / \mathrm{K} 149 \mathrm{~A}(\mathrm{M} 13)$ \\
\hline pMZ2688 & Gag3 & $1-290$ & E190A/R191A (M18) \\
\hline pMZ2650 & Gag3 & $1-290$ & G87A (MHR2) \\
\hline pMZ2651 & Gag3 & $1-290$ & F93A (MHR4) \\
\hline pVB2832 & CA-CTD & $136-207$ & $\mathrm{E} 148 \mathrm{~A} / \mathrm{K} 149 \mathrm{~A}(\mathrm{M} 13)$ \\
\hline pVB2833 & CA-CTD & $136-207$ & E190A/R191A (M18) \\
\hline \multicolumn{4}{|c|}{ pGAD T7 (with Gal4-AD): } \\
\hline pMZ2677 & Gag3 & $1-290$ & WT \\
\hline pMZ2678 & Gag3 $\Delta S P$ & $\Delta 207-233$ & WT \\
\hline pMZ2679 & $\mathrm{p} 27$ & $1-233$ & WT \\
\hline pMZ2680 & CA & $1-207$ & WT \\
\hline pMZ2681 & CA-NTD & $1-135$ & WT \\
\hline pMZ2682 & CA-CTD & $136-207$ & WT \\
\hline pMZ2683 & NC & $234-290$ & WT \\
\hline pMZ2686 & Gag3 & $1-290$ & D60A/R63A (M4) \\
\hline pMZ2655 & Gag3 & $1-290$ & $\mathrm{E} 148 \mathrm{~A} / \mathrm{K} 149 \mathrm{~A}(\mathrm{M} 13)$ \\
\hline pMZ2689 & Gag3 & $1-290$ & E190A/R191A (M18) \\
\hline pMZ2653 & Gag3 & $1-290$ & G87A (MHR2) \\
\hline pMZ2654 & Gag3 & $1-290$ & F93A (MHR4) \\
\hline pVB2829 & CA-NTD & $1-135$ & D60A/R63A (M4) \\
\hline pVB2830 & CA-NTD & $1-135$ & G87A (MHR2) \\
\hline pVB2831 & CA-NTD & $1-135$ & F93A (MHR4) \\
\hline
\end{tabular}

$\mathrm{AD}=$ activation domain; $\mathrm{BD}=$ binding domain; $\mathrm{CA}=$ capsid; $\mathrm{CTD}=\mathrm{C}$-terminal domain; $\mathrm{NC}=$ nucleocapsid; $\mathrm{NTD}=\mathrm{N}$-terminal domain; $\mathrm{SP}=$ spacer; WT = wild type.

isolates from each transformation were restreaked and then replica plated onto complete synthetic medium lacking adenine, tryptophan and leucine and grown at $30^{\circ} \mathrm{C}$ for evaluation of color development and growth. We first tested Gag3, CA, p27, and NC fusions in both vectors in all combinations. A mutant deleted for SP residues 208 to $232(\triangle \mathrm{SP})$ was also assayed (K Christiansen, MZ, VB and $\mathrm{SBS}$, unpublished results). AD-CA transformants were not recovered as stable colony isolates in repeated attempts. Other constructs were shown to be positive for expression of the appropriate fusion by immunoblot anal- ysis using rabbit polyclonal antibodies against Gal4 $\mathrm{AD}$ and Gal4 BD (Upstate Biotechnology, Lake Placid, NY, USA) or CA [5] (data not shown) [6]. With the exception of p27, which produced a slight amount of background growth in cells with both vectors, these domains were negative for reporter activation when expressed from either vector in the presence of the other vector (Figure 1, Additional files 1, 2, 3, 4, 5, 6, 7, 8, 9, 10, 11, 12).

Homotypical interactions (except for CA) and heterotypical interactions were assayed (Figure 1). Gag3, $\Delta \mathrm{SP}$, and p27 interacted in both vector contexts and in all com- 
binations tested. BD-CA interacted with AD-Gag3, AD$\triangle \mathrm{SP}$, and AD-p27. NC fusions were expressed (data not

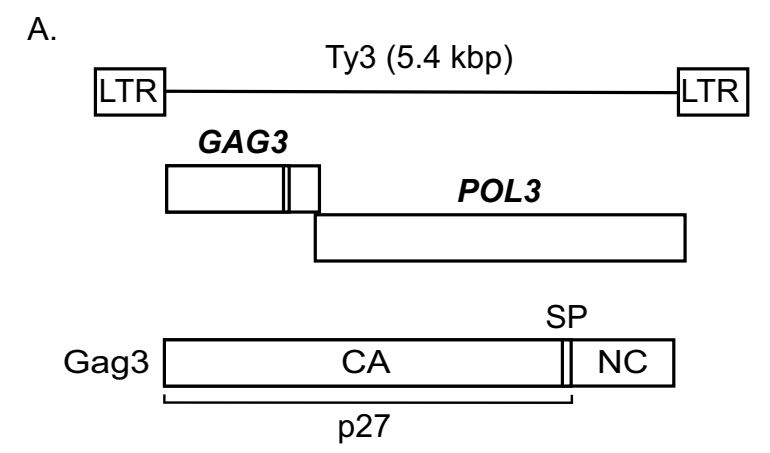

B.
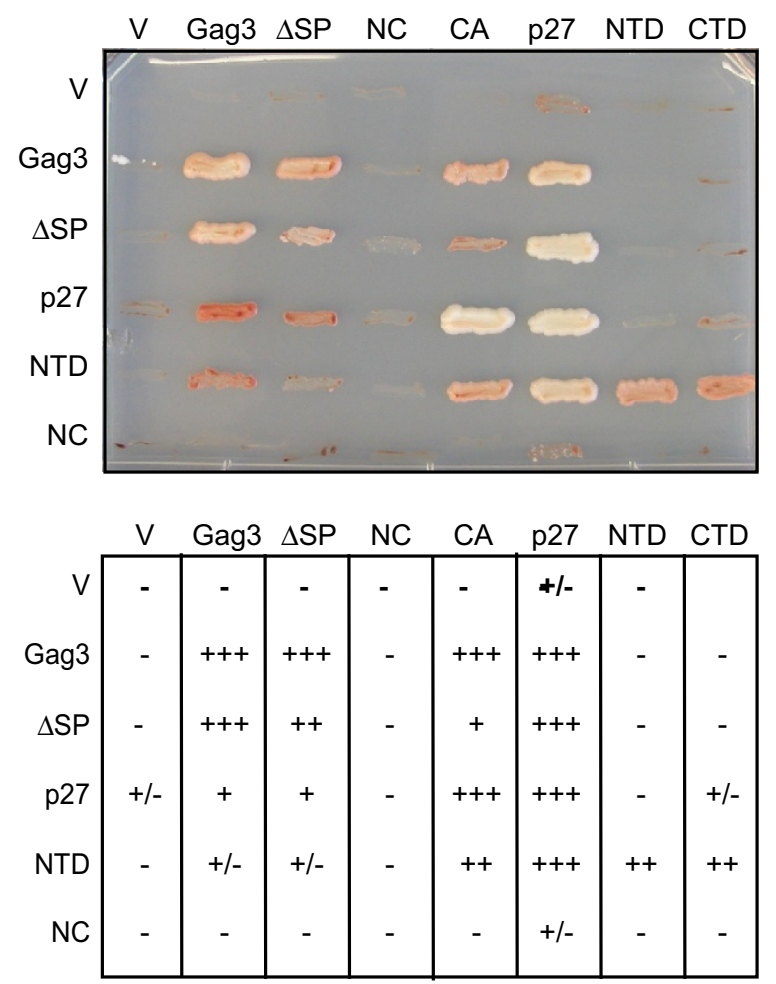

Figure 1 Interaction of Ty3 Gag3 subdomains shown by interactions between Gal4 DNA binding domain (BD) and activation domain (AD) fusions to Ty3 Gag3 subdomains. Interaction restores Gal4 mediated activation of ADE2 under control of the GAL1-10 upstream activating sequences (UASs) and growth on medium lacking adenine. Cells expressing ADE2 have reduced accumulation of a red adenine biosynthetic intermediate. Yeast strain yAH109 was trans-

formed with two-hybrid pGAD or pGBK vectors $(V)$ or expression plasmids. Streaks are representative of independent transformants growing on synthetic dextrose medium lacking tryptophan, leucine, and adenine. Gal4-BD fusions are labeled in the top row and shown in columns. Gal4-AD fusions are labeled in the left column and shown in rows. Scoring is based on four individual transformants. The most variability was observed among individual transformants expressing Gag3 and capsid (CA)-N-terminal domain fusions. shown), but failed to interact with any other fusion (Figure 1). Together these results indicated that the CA domain is capable of interactions initiated in the absence of SP and NC domains. The strong interactions of p27 with itself, compared to homotypical interactions of Gag3 or $\triangle \mathrm{SP}$ suggested that the NC domain makes a slight negative contribution.

Among the previously described alanine-scanning mutations in Ty3 Gag3 [3] were ones that appeared to disrupt particle morphogenesis at specific stages or in specific ways. We reasoned that the interactions disrupted in particular mutants could be inferred from the effects of mutations in two-hybrid interactions. The Ty3 CA NTD from amino acids 86 to 100 includes a motif similar to the retroviral major homology region (MHR) $\left(\mathrm{QGX}_{2} \mathrm{EX}_{2} \mathrm{FX}_{2} \mathrm{FX}_{3} \mathrm{~L}\right)$ [7-9]. Alanine substitutions at the second and fourth conserved positions in this motif (MHR2/G87A and MHR4/F93A) reduced VLP formation and processing [3]. Within the CA domain of Gag3, two sets of mutations, D60A/R63A and E148A/K149A, resulted in small Ty3 protein clusters, disrupted P-body component association, and blocked assembly and processing. One pair of alanine substitutions in the CA Cterminal domain (CTD), E190A/R191A, caused extensive cytoplasmic Ty3 protein filament formation, but did not disrupt association with P-body components. These mutations were introduced into the Gag3 two-hybrid system. Constructs were evaluated in all possible combinations with each other and with wild-type Gag3 (Table 2, Additional files 1, 2, 3, 4, 5, 6). Gag3 fusions containing mutations that blocked assembly failed to show homotypical interactions and heterotypic interactions among themselves. Homotypical interactions of E190A/R191A Gag3, which was associated with filament formation, were at least as strong as those of wild-type Gag3. Wildtype binding domain (BD)-Gag3 or BD-E190A/R191A showed heterotypical interactions with activation domain (AD)-D60A/R63A, AD-MHR2, AD-MHR4, and ADE148A/K149A. These results suggested that the assembly mutations act by disrupting intermolecular interfaces rather than by causing global misfolding of the Gag3 molecule.

Studies of retroviral CA assemblies show a network of CA NTD hexamers connected via CA CTD interactions (reviewed in [10]). These interactions are elucidated in more detail by studies of the crystal structures of the Moloney murine leukemia virus NTD hexamer [11] and full-length HIV1 CA [12,13]. Ty3 CA is also predicted to have CA-NTD and CA-CTD alpha helical bundles [3] and capsomeres with sixfold symmetry have been imaged on the surface of Ty3 immature and mature VLPs by atomic force microscopy, suggesting that CA NTD forms a surface hexameric network analogous to what occurs in mature retrovirus cores [2]. In order to test for the inde- 
Table 2: Interactions of Gag3 mutants

\begin{tabular}{|c|c|c|c|c|c|c|c|}
\hline & $\mathbf{V}$ & Gag3 & D60A/R63A & MHR2 & MHR4 & E148A/K149A & E190A/R191A \\
\hline $\mathrm{V}$ & - & - & - & - & - & - & - \\
\hline Gag3 & - & +++ & - & - & - & - & +++ \\
\hline D60A/R63A & - & + & - & - & - & - & ++ \\
\hline MHR2 & ND & ++ & - & - & - & - & ++ \\
\hline MHR4 & ND & ++ & - & - & - & - & ++ \\
\hline $\mathrm{E} 148 \mathrm{~A} / \mathrm{K} 149 \mathrm{~A}$ & - & ++ & - & - & - & - & ++ \\
\hline E190A/R191A & - & +++ & - & - & - & - & +++ \\
\hline
\end{tabular}

pendent contributions of the NTD and CTD to interactions occurring during VLP assembly, these domains were expressed in the two-hybrid vectors. Expression was confirmed for AD-NTD and BD-NTD, and BD-CTD. AD-CTD transformants, similar to AD-CA transformants, could not be isolated, and it was concluded that expression of this fusion was toxic. Interactions were tested between NTD and CTD and between each of them and Gag3, CA, p27, $\triangle$ SP and NC (Figure 1 and Additional file 7). Interactions between AD-NTD and BD-NTD and between AD-NTD and BD-CTD were readily detected. Interactions were marginal and variable between NTD and Gag3 and $\triangle \mathrm{SP}$, but were readily detected between AD-NTD and BD-p27 and BD-CA. These results showed that the isolated NTD and CTD interact and that the target of the interaction, in the case of the NTD, is less accessible in the precursor Gag3 and $\triangle \mathrm{SP}$ than in processing products $\mathrm{p} 27$ and $\mathrm{CA}$.

In order to test whether mutations in Gag3 that affected assembly and homotypical and heterotypical interactions of intact Gag3 changed the exposure of NTD and CTD subdomains and therefore their ability to interact, interaction of these subdomains with mutant Gag3 was examined (Table 3, Additional files 8 and 9). No mutation in AD-Gag3 enhanced the ability of Gag3 to interact with BD-NTD or allowed it to interact with BDCTD, suggesting that these mutations did not disrupt assembly by causing premature exposure of subdomain interaction sites. In order to determine which, if any, of the subdomain interactions might be directly affected by these mutations, the mutations were also introduced into the subdomains. Because AD-CTD was toxic and the BD-
NTD reacted with fewer partners than AD-NTD, the mutations were introduced into BD-CTD and AD-NTD. Introduction of assembly mutations MHR2 and MHR4 into the NTD slightly weakened Gag3 AD-NTD interaction (Tables 3, 4, Additional file 10), and introduction of D60A/R63A further diminished the interaction. The effect of mutations on NTD-NTD and NTD-CTD interactions were also evaluated (Table 4, Additional files 11 and 12). Although the D60A/R63A mutation had a negative effect on NTD interactions, surprisingly, MHR2 and MHR4 mutations slightly improved interactions between NTD domains. All three mutations in the AD-NTD context enhanced interactions with the CTD. Introduction of E148A/K149A or E190A/R191A mutations into BD-CTD also did not significantly affect interaction with wild-type or mutant NTDs. Thus, overall the effect of mutations that disrupt assembly was most noticeable at the level of Gag3 polyprotein interactions. The E190A/R191A mutation, which occurs close to the end of the mature $\mathrm{CA}$ and causes filamentation [3], had no effect or slightly increased interaction. The evidence that interaction is not disrupted, coupled with the highly-ordered mutant structure suggests that the mutation could have a kinetic effect on assembly, thereby trapping Gag3 in an aberrant multimeric form.

Two-hybrid analysis readily detected interactions between Gag3 polyproteins. Nevertheless there were asymmetries in the interactions introduced by the expression context. These included apparent toxicity of $\mathrm{AD}-\mathrm{CA}$ and $\mathrm{AD}-\mathrm{CTD}$, detection of stronger interactions of BD-P27 compared to AD-P27, and of AD-NTD compared to BD-NTD (Figure 1). This could be explained by 
Table 3: Effects of mutations in Gag3 on NTD and CTD interactions

\begin{tabular}{|c|c|c|c|c|}
\hline & $\mathbf{v}$ & Gag3 & NTD & CTD \\
\hline V & - & - & - & - \\
\hline Gag3 & - & +++ & - & - \\
\hline NTD & - & $+/-$ & ++ & ++ \\
\hline D60A/R63A & - & + & - & - \\
\hline MHR2 & - & ++ & - & - \\
\hline MHR4 & - & ++ & - & - \\
\hline E148A/K149A & - & ++ & - & - \\
\hline E190A/R191A & - & +++ & - & - \\
\hline
\end{tabular}

For images, see Additional files 7, 8, 9.

Abbreviations as in Table 2

context-dependent exposure of interaction domains or even by context effects on nuclear entry. Thus, the positive interactions coupled with the effects of mutations or deletions on those interactions rather than the lack of interaction in one or the other expression context offer the most meaningful outcomes of our study. Two-hybrid assays showed that the CA domain interacts independent of the NC domain. This is in contrast to the apparent situation with some retroviruses in which interactions are dependent upon NC subdomains [14-16]. We speculate that this interaction contributes to the distinctive stability of the Ty3 particle. Examination of $\Delta \mathrm{SP}, \mathrm{p} 27$ and CA showed that interactions comparable to those between Gag3 polyproteins occur in the absence of SP and NC. This is consistent with other studies that show that defective Ty3 multimers form in the absence of these domains $[17,18]$ (SBS and K Christiansen, University of CA, Irvine, unpublished results).

Recent 3D structures of full-length CA molecules in assembled HIV1 particles viewed as 2D crystals by cryoelectron microscopy (cryoEM) underscore three types of interactions with retroviral CA assembled into hexameric lattice structures: NTD-NTD and CTD-NTD intermolecular interactions and CTD-CTD interhexameric inter-

Table 4: Effects of mutations on NTD and CTD interactions

\begin{tabular}{|c|c|c|c|c|c|c|}
\hline & $\mathbf{V}$ & Gag3 & NTD & CTD & CTD E148A/K149A & CTD E190A/R191A \\
\hline V & - & - & - & - & - & - \\
\hline Gag3 & - & +++ & - & - & - & - \\
\hline NTD & - & $+/-$ & ++ & ++ & ++ & ++ \\
\hline NTD D60A/R63A & - & - & + & +++ & ++ & ++ \\
\hline NTD MHR2 & - & $+/-$ & +++ & +++ & +++ & +++ \\
\hline NTD MHR4 & - & $+/-$ & +++ & +++ & +++ & +++ \\
\hline
\end{tabular}

For images, see Additional files 10, 11, 12.

Abbreviations as in Table 2 
actions [12,19]. NTD-NTD interactions and NTD-CTD interactions are also supported by EM studies of in vitro assembly intermediates of RSV [20]. Based on the similar 3D structures of different retroviral CA proteins, these interactions are believed to be generally conserved among retroviruses. Our data are consistent with the existence of CA NTD-NTD interactions and CTD-NTD interactions within the Ty3 VLPs, similar to what is observed in the HIV1 CA 2D crystal structure and in the RSV cryoEM. The fact that these interactions are observed for isolated subdomains and between NTD and CA suggests that they underlie the stability of the mature Ty3 particle.

Finally, this investigation provides further support for our model that Gag3 undergoes conformational switching between unassembled and assembled states [17]. First, isolated NTD interacted preferentially with p27 and CA, compared to Gag3, indicating that the NTD interface may become more accessible during maturation. Second, the CTD failed to interact with Gag3, but interacted readily with the isolated NTD. Third, mutations that blocked native assembly had their most pronounced effect at the level of Gag3, rather than in individual domain interactions, suggesting that exposure of interaction surfaces is not equivalent in Gag3 and intermediate forms. It is also possible that the subdomain interactions play a role in the hypothesized conformational switch: Intramolecular NTD-CTD interactions might help to order assembly by limiting NTD-NTD interactions until precursor localization or RNA association is achieved. Similarly, NC, the presence of which appears to correlate with decreased interaction between Gag3 derivatives and NTD, might help to limit completion of intermolecular interactions until genomic RNA is engaged. Our findings also pose the possibility that two-hybrid assays of retroviral CA subdomains might provide detection of interactions that have thus far been detected in only in crystallographic studies. If successful, such an assay could be useful in high throughput screening for inhibitors of assembly.

\section{Additional material}

Additional file 1 Sup. Fig. 1. Interactions of BD M4 mutant Gag3 D60A/R63A with Gag3 wild type, D60A/R63A, G87A, F93A, E148A/ K149A, and E190A/R191A. The D60A/R63A mutation disrupts interactions with wild type and other mutant Gag3 proteins. Images of yeast strain yAH109 containing two-hybrid pGAD or pGBK vectors or expression plasmids as described in text. Cells are shown as representative streaks of independent transformants growing on synthetic dextrose medium lacking tryptophan, leucine, and adenine. Mutants are M4 (D60A/R63A), MHR2 (G87A), MHR4 (F93A), M13 (E148A/K149A), and M18 (E190A/R191A). Binding domain (BD) fusions are labeled in the top row and shown in columns; activation domain (AD) fusions are labeled in the left column and shown in rows. Scoring is based on four individual transformants (two shown). There was variability among individual BD-Gag3/AD-N-terminal domain (NTD) transformants (for example Figure 1, Additional file 7 and Additional file 10).
Additional file 2 Sup. Fig. 2. Interactions of BD MHR2 mutant Gag3 G87A with Gag3 wild type, G87A, F93A, E148A/K149A, and E190A/ R191A. The G87A mutation disrupts interactions with wild type and other mutant Gag3 proteins.

Additional file 3 Sup. Fig. 3. Interactions of BD MHR4 mutant Gag3 F93A with Gag3 wild type, F93A, G87A, E148A/K149A, and E190A/ R191A. The F93A mutation disrupts interactions with wild type and other mutant Gag3 proteins.

Additional file 4 Sup. Fig. 4. Interactions of BD M13 mutant Gag3 E148A/K149A with Gag3 wild type, E148A/K149A, D60A/R63A, G87A, F93A, and E190A/R191A. The E148A/K149A mutation disrupts interactions with wild type and other mutant Gag3 proteins.

Additional file 5 Sup. Fig. 5. Interactions of BD M18 mutant Gag3 E190A/R191A with Gag3 wild type, E190A/R191A, D60A/R63A, G87A, F93A, and E148A/K149A. BD E190A/R191A interacts with other mutant Gag3 proteins, although less well with D60A/R63A, G87A, and F93A.

Additional file 6 Sup. Fig. 6. Interactions of BD wild type Gag3 with wild type Gag3, D60A/R63A, G87A, F93A, E148A/K149A, and E190A/ R191A. Wt Gag3 interacts with other mutant Gag3 proteins, although much less well with D60A/R63A, G87A, and F93A.

Additional file 7 Sup. Fig. 7. Interactions of BD capsid (CA) NTD with wild type Gag3, CA NTD, and CA CTD. BD CA NTD interacts with CA NTD and CA CTD.

Additional file 8 Sup. Fig. 8. BD capsid (CA) NTD interaction with Gag3 wild type and D60A/R63A, G87A, F93A, E148A/K149A, and E190A/ R191A. Mutations in the CA NTD and CA CTD that disrupt interactions in the Gag3 context fail to allow observation of interactions between CA NTD and Gag3.

Additional file 9 Sup. Fig. 9. Interactions between BD capsid (CA) CTD and wild type Gag3, D60A/R63A, G87A, F93A E148A/K149A, and E190A/R191A. Mutations in Gag3 that disrupt Gag3 interactions in the Gag3 context fail to allow observation of interactions between CA CTD and Gag3.

Additional file 10 Sup. Fig. 10. Interactions between BD Gag3 and wild type Gag3, wild type CA NTD, and capsid (CA) NTD D60A/R63A, CA NTD G87A, and CA NTD F93A. Mutations in the CA NTD that disrup interactions in the Gag3 context fail to allow observation of interactions between CA NTD and Gag3.

Additional file 11 Sup. Fig. 11. Interactions between BD capsid (CA) NTD and BD CA CTD with wild type CA NTD, CA NTD D60A/R63A, CA NTD G87A, and CA NTD F93A. With the exception of D60A/R63A mutations in the CA NTD that disrupt Gag3 interactions do not decrease CA NTD interactions with the CA NTD. D60A/R63A does not decrease interactions with BD CA CTD.

Additional file 12 Sup. Fig. 12. Interactions between BD capsid (CA) CTD M13 mutant E148A/K149A and BD CA CTD M18 mutant E190A/ R191A with wild type Gag3, CA NTD, CA NTD D60A/R63A, CA NTD G87A, and CA NTD F93A. Mutations in CA CTD and CA NTD that disrupt Gag3 interactions do not interfere with observation of interactions between the CA CTD and CA NTD.

\section{Competing interests}

The authors declare that they have no competing interests.

\section{Authors' contributions}

$M Z$ and SBS designed the study and drafted the manuscript. MZ and LZ designed and constructed Ty3 Gag3 mutant collection. MZ and VB subcloned into two-hybrid vectors and together with BI performed the two-hybrid analysis. All authors read and approved the manuscript.

\section{Acknowledgements}

This research was supported in part by funds from the National Institutes of Health including Public Health Services grant GM33281 to SBS.

\section{Author Details}

1Department of Biological Chemistry, University of California, Irvine, CA, USA, 2Department of Microbiology and Molecular Genetics, University of California, Irvine, CA, USA and ${ }^{3}$ Institute for Genomics and Bioinformatics, University of California, Irvine, CA, USA 


\section{References}

1. Sandmeyer SB, Aye M, Menees TM: Ty3: a position-specific, gypsylike element in Saccharomyces cerevisiae. In Mobile DNA I/ Edited by: Craig NL, Craigie R, Gellert M, Lambowitz AM. Washington DC: ASM Press; 2002:663-682.

2. Kuznetsov YG, Zhang M, Menees TM, McPherson A, Sandmeyer S: Investigation by atomic force microscopy of the structure of Ty3 retrotransposon particles. J Virol 2005, 79:8032-8045.

3. Larsen LS, Zhang M, Beliakova-Bethell N, Bilanchone V, Lamsa A, Nagashima K, Najdi R, Kosaka K, Kovacevic V, Cheng J, Baldi P, Hatfield GW, Sandmeyer S: Ty 3 capsid mutations reveal early and late functions of the amino-terminal domain. J Virol 2007, 81:6957-6972.

4. Fields S, Song O: A novel genetic system to detect protein-protein interactions. Nature 1989, 340:245-246.

5. Zhang M: Ty3 virus-like particle morphogenesis and assembly. In Thesis University of California, Irvine; 2007.

6. Menees TM, Sandmeyer SB: Transposition of the yeast retroviruslike element Ty3 is dependent on the cell cycle. Mol Cell Biol 1994, 14:8229-8240

7. Orlinsky KJ, Gu J, Hoyt M, Sandmeyer S, Menees TM: Mutations in the Ty3 major homology region affect multiple steps in Ty3 retrotransposition. J Virol 1996, 70:3440-3448.

8. Ivanov D, Tsodikov OV, Kasanov J, Ellenberger T, Wagner G, Collins T: Domain-swapped dimerization of the HIV-1 capsid C-terminal domain. Proc Natl Acad Sci USA 2007, 104:4353-4358.

9. Craven RC, Leure-du Pree AE, Weldon RA Jr, Wills JW: Genetic analysis of the major homology region of the Rous sarcoma virus Gag protein. $J$ Virol 1995, 69:4213-4227.

10. Sundquist WI, Hill CP: How to assemble a capsid. Cell 2007, 131:17-19.

11. Mortuza GB, Haire LF, Stevens A, Smerdon SJ, Stoye JP, Taylor IA: Highresolution structure of a retroviral capsid hexameric amino-terminal domain. Nature 2004, 431:481-485.

12. Pornillos O, Ganser-Pornillos BK, Kelly BN, Hua Y, Whitby FG, Stout CD, Sundquist $\mathrm{WI}$, Hill $C P$, Yeager $\mathrm{M}$ : $\mathrm{X}$-ray structures of the hexameric building block of the HIV capsid. Cell 2009, 137:1282-1292.

13. Ganser-Pornillos BK, Cheng A, Yeager M: Structure of full-length HIV-1 CA: a model for the mature capsid lattice. Cell 2007, 131:70-79.

14. Bowzard JB, Bennett RP, Krishna NK, Ernst SM, Rein A, Wills JW: Importance of basic residues in the nucleocapsid sequence for retrovirus Gag assembly and complementation rescue. J Virol 1998, 72:9034-9044

15. Scarlata S, Carter C: Role of HIV-1 Gag domains in viral assembly. Biochim Biophys Acta 2003, 1614:62-72.

16. Zabransky A, Hunter E, Sakalian M: Identification of a minimal HIV-1 gag domain sufficient for self-association. Virology 2002, 294:141-150.

17. Larsen LS, Beliakova-Bethell N, Bilanchone V, Zhang M, Lamsa A, Dasilva R, Hatfield GW, Nagashima K, Sandmeyer S: Ty3 nucleocapsid controls localization of particle assembly. J Virol 2008, 82:2501-2514.

18. Larsen LS, Kuznetsov Y, McPherson A, Hatfield GW, Sandmeyer S: TY3 GAG3 protein forms ordered particles in Escherichia coli. Virology 2008, 370:223-227.

19. Ganser-Pornillos BK, Yeager M, Sundquist WI: The structural biology of HIV assembly. Curr Opin Struct Bio/ 2008, 18:203-217.

20. Purdy JG, Flanagan JM, Ropson IJ, Craven RC: Retroviral capsid assembly: a role for the CA dimer in initiation. J Mol Biol 2009, 389:438-451.

doi: 10.1186/1759-8753-1-14

Cite this article as: Zhang et al., Two-hybrid analysis of Ty3 capsid subdomain interactions Mobile DNA 2010, 1:14

\section{Submit your next manuscript to BioMed Central} and take full advantage of:

- Convenient online submission

- Thorough peer review

- No space constraints or color figure charges

- Immediate publication on acceptance

- Inclusion in PubMed, CAS, Scopus and Google Scholar

- Research which is freely available for redistribution

Submit your manuscript at www.biomedcentral.com/submit
C Biomed Central 